\title{
2-Aminoethoxydiphenyl borate activates the mechanically gated human KCNK channels KCNK 2 (TREK-1), KCNK 4 (TRAAK), and KCNK 10 (TREK-2)
}

\author{
Leopoldo Beltrán*, Madeline Beltrán, Ainhara Aguado, Günter Gisselmann and Hanns Hatt
}

Department of Cell Physiology, Ruhr-University-Bochum, Bochum, Germany

Edited by:

Nick Andrews, Harvard Medical

School, USA

\section{Reviewed by:}

Enrico Sanna, University of Cagliari, Italy

Teresa Giraldez, University Hospital NS Candelaria, Spain

\section{*Correspondence:}

Leopoldo Beltrán, Department of Cell Physiology, Ruhr-Universität Bochum, ND 4-132, Universitaetsstraße 150, 44780 Bochum, Germany. e-mail: leopoldo.beltran@rub.de
Two-pore domain $\mathrm{K}^{+}\left(\mathrm{KCNK}, \mathrm{K}_{2} \mathrm{P}\right)$ channels underlie the "leak" (background) potassium conductance in many types of excitable cells. They oppose membrane depolarization and cell excitability. These channels have been reported to be modulated by several physical and chemical stimuli. The compound 2-aminoethoxydiphenyl borate (2-APB) was originally described as an inhibitor of $\mathrm{IP}_{3}$-induced $\mathrm{Ca}^{2+}$ release but has been shown to act as either a blocker or an activator for several ion channels. Here, we report the effects of this compound on members of the TREK (TWIK related $\mathrm{K}^{+}$channels) subfamily of human KCNK channels. We injected Xenopus laevis oocytes with cRNAs (complementary RNAs) encoding several KCNK channels and measured their response using the two-electrode voltage clamp technique. 2-APB was found to be an effective activator for all members of the TREK subfamily (hKCNK2, hKCNK4, and hKCNK10), with the highest efficacy in hKCNK10. We also found that 2-APB was able to activate these channels in cell-excised patches of HEK293 (human embryonic kidney 293) cell transfected with hKCNK4 or hKCNK10, demonstrating direct activation. TREK channels are widely expressed in the central nervous system and peripheral tissues, where they play roles in several key processes. However, little is known regarding their pharmacology; therefore, the identification of a common, stable and inexpensive agonist should aid further investigations of these channels. Additionally, 2-APB has been used to study native receptors in cell systems that endogenously express members of the TREK subfamily (e.g., rat dorsal root ganglia); our results thus warn against the use of 2-APB at high concentrations in these systems.

\section{Keywords: 2-APB, KCNK channels, TREK, Xenopus oocytes, two-electrode voltage clamp}

\section{INTRODUCTION}

KCNK channels (also known as $\mathrm{K}_{2 \mathrm{P}}$, for two-pore domain potassium channels) are potassium selective channels that tend to be constitutively open. Most of them behave as outward rectifiers under physiological $\mathrm{K}^{+}$concentrations. Additionally, they also behave in an almost voltage-independent manner (Enyedi and Czirják, 2010). Because of these characteristics, they are considered the main channels responsible for the leak potassium current that helps maintain the resting membrane potential. This leak current exerts control over neuronal excitability by shaping the duration, frequency, and amplitude of action potentials (Goldstein et al., 2001). An increased $\mathrm{K}^{+}$leak current stabilizes the cell at hyperpolarized voltages below the firing threshold, whereas leak suppression permits or facilitates depolarization. In this manner, KCNK channels play a role in such diverse processes as metabolic regulation, apoptosis, thermoperception, and chemoperception (Lotshaw, 2007).

The mechanically gated TREK (for TWIK related $\mathrm{K}^{+}$channels) subfamily of KCNK channels is composed of three members: TREK-1 (KCNK2), TRAAK (KCNK4), and TREK-2 (KCNK10) with several functional splice variants reported for hTREK-1 and hTREK-2 (Gu et al., 2002; Xian Tao et al., 2006). Although members of the TREK subfamily differ slightly in their electrophysiological properties, they show similar characteristics, such as mechano- and thermosensitivity. They also present overlapping pharmacological patterns, characterized by a mild sensitivity to standard potassium channel blockers such as quinidine and $\mathrm{Ba}^{2+}$ and sensitivity to volatile anesthetics like halothane (Lotshaw, 2007). TREK channels are widely expressed in both the peripheral and the central nervous system (CNS; Medhurst et al., 2001). Recent studies with TREK ${ }^{-1-}$ mice have demonstrated that TREK channels are necessary for appropriate mechano- and thermoperception (Alloui et al., 2006; Noël et al., 2009).

2-Aminoethoxydiphenyl borate (2-APB) was originally described as a membrane-permeable modulator of $\operatorname{Ins}(1,4,5) \mathrm{P}_{3}$ induced $\mathrm{Ca}^{2+}$ release (Maruyama etal., 1997). Although this function has been questioned, 2-APB is agreed to inhibit the

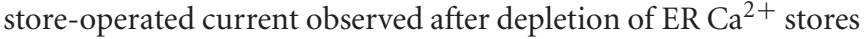
(Dobrydneva and Blackmore, 2001); it also blocks certain transient receptor potential (TRP) channels belonging to the canonical (TRPC), and melastatin (TRPM) subfamilies (Hu et al., 2004) and certain voltage-gated potassium channels present in Limulus ventral photoreceptors (Wang et al., 2002). It is also a common activator for the TRPV1 (transient receptor potential vanilloid 1), TRPV2, and TRPV3 channels (Hu et al., 2004). Interestingly, the perception of noxious heat, in which TRPV1 and TRPV2 play 
a key role, has been shown to be modulated or fine-tuned by KCNK2 and KCNK4 (Noël et al., 2009). Both the TREK and TRPVs subfamilies have been shown to be coexpressed at the neuronal level in the rat trigeminal ganglia (Yamamoto et al., 2009). With this background in mind, we addressed the question of whether 2-APB has an effect on human KCNK channels of the TREK subfamily. We found that 2-APB activates all members of this subfamily in a dose-dependent manner and has the greatest effect on hKCNK10.

\section{EXPERIMENTAL PROCEDURES MOLECULAR BIOLOGY}

hKCNK4 cloned into pBluescript SK+, hCKNK10 cloned into pCR4-TOPO and hKCNK2 cloned into pDNR-dual were purchased from Imagenes (Berlin, Germany). hKCNK2 and hKCNK10 cDNA inserts were afterward subcloned into pSGEM for electrophysiological recordings in Xenopus oocytes. The plasmids were linearized with NotI for pBluescript SK+ and PacI for pSGEM, providing linear cDNA templates for in vitro transcription. All three hKCNK inserts were subsequently subcloned into pCDNA3 for electrophysiological recordings in human embryonic kidney 293 (HEK293) cells.

hKCNK3 cloned into the expression vector pIRES-CD8 was a gift from Dr. Fabrice Duprat (Institut National de la Santé et de la Recherche Médicale, Antipolis, France). hKCNK18 cloned into the plasmid pEXO was a gift from Professor Dr. P. Enyedi (Semmelweis University, Budapest, Hungary). hKNCK9 cloned into the expression vector pCR4-TOP, was purchased from Imagenes (Berlin, Germany). The plasmids were linearized with the restriction enzymes BamHI, XhoI, and SspI, respectively, and then used as templates for in vitro transcription.

Capped RNAs were synthesized in the presence of capping ana$\log \mathrm{m} 7 \mathrm{G}\left(5^{\prime}\right) \mathrm{ppp}\left(5^{\prime}\right) \mathrm{G}$ using the AmpliCap-T7 Message Maker kit (Epicentre, Madison, WI, USA). Complementary RNA (cRNA) was dissolved in nuclease-free water to give a final concentration of $500 \mathrm{ng} / \mu \mathrm{L}$.

\section{Xenopus laevis OOCYTE PREPARATION}

Complementary RNAs were expressed in X. laevis oocytes essentially as described by Vogt-Eisele et al. (2007). Briefly, mature female $X$. laevis frogs were anesthetized with $0.15 \%$ MS-222, surgery was performed according to standard methods, and the extracted ovarian lobes were placed into $\mathrm{Ca}^{2+}$-free Barth's solution containing collagenase type II (Worthington Biochemical Corporation) and incubated for $90 \mathrm{~min}$ on a shaker at $40 \mathrm{rpm}$ (room temperature). After that time, healthy stage $\mathrm{V}$ and $\mathrm{VI}$ oocytes were selected for injection with cRNA coding for the proteins of interest; $30 \mathrm{~nL}$ of cRNA $(100 \mathrm{ng} / \mu \mathrm{L})$ for each channel was injected using a Microinjector Micro 4 TM (World Precision Instruments). After injection, the oocytes were incubated in ND96 $\left(96 \mathrm{mM} \mathrm{NaCl} ; 2 \mathrm{mM} \mathrm{KCl} ; 1.8 \mathrm{mM} \mathrm{CaCl}_{2} ; 1 \mathrm{mM}\right.$ $\mathrm{MgCl}_{2} ; 2.5 \mathrm{mM}$ sodium pyruvate $3 \mathrm{mM}$, 4-(2-hydroxyethyl)-1-

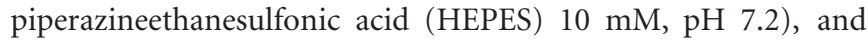
measurements were performed 24-72 h after injection.

The oocytes were placed in a $100 \mu \mathrm{L}$ chamber perfused with frog Ringer's solution (115 mM NaCl; $2.5 \mathrm{mM} \mathrm{KCl} ; 1.8 \mathrm{mM}$ $\mathrm{CaCl}_{2}$, and $10 \mathrm{mM}$ HEPES $\mathrm{pH}$ 7.2); this solution was also used to dissolve the applied chemicals. For recording, a Turbo Tec-03x (npi electronic $\mathrm{GmbH}$ ) amplifier was used. Borosilicate glass capillaries were pulled with a Kopf vertical pipette puller. For the voltage clamp, the pulled glass capillaries were filled with $3 \mathrm{M} \mathrm{KCl}$.

\section{TWO-ELECTRODE VOLTAGE CLAMP MEASUREMENTS}

A ramp series protocol was used to evaluate the activity of the channels. The voltage ramps consisted of a starting constant voltage of $-100 \mathrm{mV}$ for $300 \mathrm{~ms}$, followed by a ramp to $+50 \mathrm{mV}$ in $700 \mathrm{~ms}$, then a constant voltage at $+50 \mathrm{mV}$ for $300 \mathrm{~ms}$, and a final constant voltage at $-40 \mathrm{mV}$ for $300 \mathrm{~ms}$. The time interval between the ramps was of $2 \mathrm{~s}$. In order to evaluate the effect of a substance on the basal current, we took the average of the currents registered during the last $30 \mathrm{~ms}$ of the $+50 \mathrm{mV}$ constant from the three ramps that showed the maximal response to the applied substance and divided it by the average obtained from three ramps prior to the application.

The data were collected using the Cellworks Reader 3.7 software (npi instruments, Germany) and analyzed with Clampfit v10.2.0.14 (MDS Analytical Technologies). Curve fitting was performed using the Hill equation (Sigmaplot 8, Systat software inc.). Data are expressed as means \pm SEM (standard error of the mean). A paired $t$-test was used to evaluate the statistical significance of the results, and $P<0.05$ was considered statistically significant and is marked with one star, $P<0.01$ is marked with two stars, and $P<0.001$ is marked with three stars.

\section{CELL CULTURE}

Human embryonic kidney 293 cells were grown in Dulbecco's modified Eagle's medium containing 10\% fetal bovine serum, $2 \mathrm{mM}$ L-glutamine, and $100 \mu \mathrm{g} / \mathrm{mL}$ penicillin/streptomycin (Invitrogen, Karlsruhe, Germany) at $37^{\circ} \mathrm{C}$ in a humidity-controlled incubator with $5 \% \mathrm{CO}_{2}$.

\section{TRANSIENT EXPRESSION OF HUMAN KCNK2, KCNK4, AND KCNK10 IN HEK293 CELLS}

For transient expression of human KCNK2, KCNK4, and KCNK10 in HEK293 cells, we used the recombinant expression plasmid pCDNA3. HEK293 cells were transiently transfected $(8 \mu \mathrm{g}$ of hKCNK4 cDNA in pCDNA3 per dish) in 35-mm dishes (Falcon, BD Bioscience, Heidelberg, Germany) using the CaP-precipitation method described previously by Dörner et al. (2007). These cells were cotransfected with pIRES-EGFP ( $1 \mu$ g per dish), which served as a transfection marker. All recordings were performed 18-24 h after transfection.

\section{ELECTROPHYSIOLOGY IN HEK293 CELLS}

Recordings were performed using the whole-cell and inside-out modes of the patch-clamp technique. Cells were maintained in an extracellular recording solution containing (in mM) $140 \mathrm{NaCl}$, $5 \mathrm{KCl}, 2 \mathrm{MgCl}_{2}, 2 \mathrm{CaCl}_{2}, 10$ HEPES, and 10 glucose, $\mathrm{pH}$ 7.4. Patch electrodes were pulled from borosilicate glass $(1.2 \mathrm{~mm}$ $\mathrm{OD} \times 1.17 \mathrm{~mm}$ ID; Harvard apparatus, Edenbridge, Kent, UK) and fire polished to 4-6 M $\Omega$ tip resistance using a horizontal pipette puller (Zeitz Instruments, Munich, Germany) in order to obtain a patch of approximately $1-2 \mu \mathrm{m}$ diameter. 


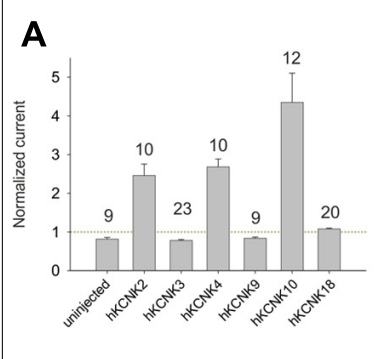

B

C

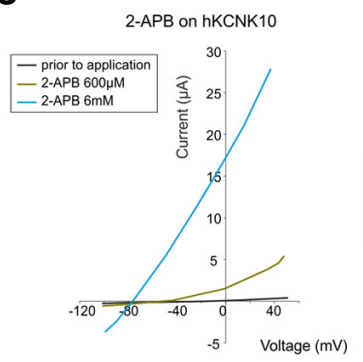

\section{D}

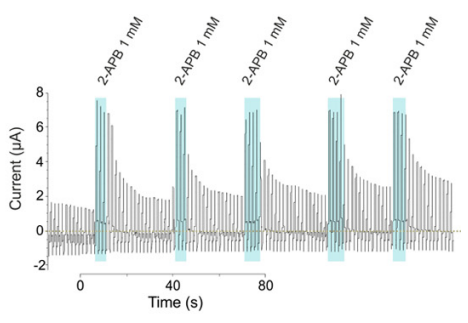

E

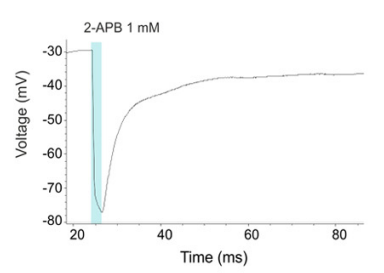

$\mathbf{F}$

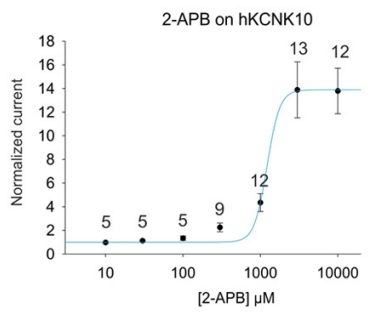

G
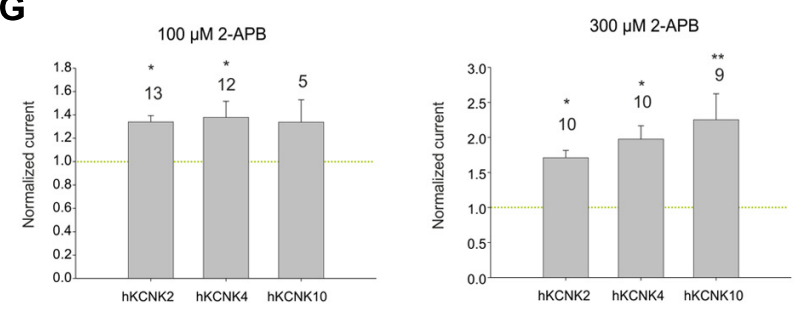

H

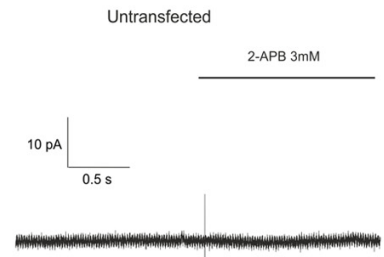

hKCNK10 transfected HEK cells

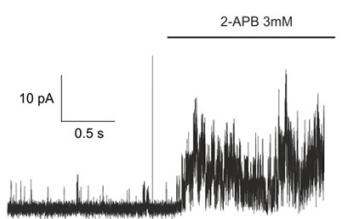

FIGURE 1 | (A) Effect of $1 \mathrm{mM}$ 2-APB on (from left to right) uninjected Xenopus oocytes and oocytes injected with cRNA coding for hKCNK2, hKCNK3, hKNCK4, hKCNK9, hKCNK10, and hKCNK18. For each group, the current registered at the final $50 \mathrm{~ms}$ of the $+50 \mathrm{mV}$ constant (see experimental procedures for a description of the ramp protocol used) was normalized to the current registered prior to the application of 2-APB (dotted line). All data are expressed as means \pm SEM. Number of cells in each experiment is indicated above the bars. (B) Absolute currents registered in uninjected Xenopus oocytes and oocytes injected with cRNA coding for hKCNK2, hKCNK4, and hKNCK10, before and after the

(Continued)

\section{Figure 1 | Continued}

application of $1 \mathrm{mM}$ 2-APB. (C) Representative I-V trace of a Xenopus oocyte expressing hKCNK10 before (black line), and after the application of 2-APB at $600 \mu \mathrm{M}$ (green line), and $6 \mathrm{mM}$ (blue line). (D) Ramp series measurement of a Xenopus oocyte expressing hKCNK10 that was repeatedly exposed to $1 \mathrm{mM}$ 2-APB. The dotted green line represents zero current. (E) $1 \mathrm{mM}$ 2-APB reversibly hyperpolarizes a Xenopus oocyte expressing hKCNK10. (F) Dose-response curve fitted to the 4-parameter Hill equation ( $n$ indicated above the points). (G) Effect of 2-APB at $100 \mu \mathrm{M}$ (left) and $300 \mu \mathrm{M}$ (right) on Xenopus oocytes injected with cRNA coding for hKCNK2, hKNCK4, and hKCNK10. Data were normalized to the current registered prior to the application of 2-APB (dotted line). All data are expressed as means \pm SEM. Number of cells in each experiment is indicated above the bars. (H) Inside-out patch-clamp recordings of cell-excised patches from untransfected HEK293 cells (left) and HEK293 cells transfected with hKCNK10 (right) before and after exposure to $3 \mathrm{mM}$ 2-APB.

For recordings, the pipette solution contained (in mM) 140 $\mathrm{KCl}, 1 \mathrm{MgCl}_{2}$, and $10 \mathrm{HEPES}, \mathrm{pH}$ 7.4. All recordings were performed at room temperature with an EPC7 amplifier (List-Medical Electronic, Darmstadt, Germany). For whole-cell patch-clamp recordings, a step protocol consisting of a holding potential of $-60 \mathrm{mV}$, followed by $20 \mathrm{mV}$ steps of $400 \mathrm{~ms}$ duration from $-110 \mathrm{mV}$ to $+50 \mathrm{mV}$ was used. Because the current registered at the last two steps continued to increase and never presented a stable baseline, the last $50 \mathrm{~ms}$ of the $+10 \mathrm{mV}$ step were selected in order to build a dose-response curve. Cell-excised patches were evaluated in the inside-out configuration, at a holding potential of $0 \mathrm{mV}$. Data were acquired using Pulse software (HEKA, Lambrecht, Germany). All data are expressed as means \pm SEM.

\section{CHEMICALS}

2-Aminoethoxydiphenyl borate and dimethyl sulfoxide (DMSO) were purchased from Sigma-Aldrich (Munich, Germany). Stock solutions of $100 \mathrm{mM}$ and $300 \mathrm{mM}$ 2-APB diluted in DMSO were made and stored at $-20^{\circ} \mathrm{C}$; the latter was used for preparing solutions containing 3 and $10 \mathrm{mM}$ 2-APB.

\section{RESULTS}

\section{2-APB ACTIVATES ALL MEMBERS OF THE TREK SUBFAMILY}

First, we studied the effect of $1 \mathrm{mM} 2-\mathrm{APB}$ on $X$. laevis oocytes injected with cRNAs coding for each member of the TREK subfamily (KCNK2, KCNK4, and KCNK10), two members of the TASK subfamily (KCNK3 and KCNK9), the only member of the TRESK subfamily (KCNK18) and a control group of noninjected oocytes (see Figure 1A). Oocytes injected with KCNK18 were not affected by 2-APB, whereas those injected with either member of the TASK subfamily were mildly inhibited by 2-APB. The currents presented by uninjected oocytes were negligible in comparison with the currents of oocytes injected with any of the aforementioned hKCNK channels; however, these currents were mildly but significantly inhibited by $1 \mathrm{mM} 2-\mathrm{APB}$, reducing their basal activity to $70.3 \%$ (Figure $1 B$ ). 2-APB, however, increased the basal activity of hKCNK2, hKCNK4, and hKCNK10 by factors of $2.22 \pm 0.2,2.68 \pm 0.2$, and $4.35 \pm 0.8$, respectively. 


\section{2-APB ACTIVATES hKCNK10 CHANNELS IN A DOSE-DEPENDENT MANNER}

We then proceeded to study in detail the effect of 2-APB on hKCNK10. hKCNK10, also known as TREK-2, is expressed throughout the human CNS. It is one of the major background $\mathrm{K}^{+}$ channels in dorsal root ganglion neurons (Kang and Kim, 2006), and it is also expressed in the kidneys, spleen, and small intestine (Medhurst et al., 2001). 2-APB produced a dose-dependent (Figure 1C) and reversible activation of hKCNK10 that was unaltered after repeated applications (Figure 1D). Consequently with this, when we recorded the membrane potential of oocytes expressing hKCNK10, we observed that $1 \mathrm{mM} 2$-APB produced a strong and quick hyperpolarizing effect that reversed almost completely after washout (Figure 1E). The dose-response curve gave an $\mathrm{EC}_{50}$ of $1.22 \pm 0.39 \mathrm{mM}$; the maximal effect (seen at around $3 \mathrm{mM}$ ) was a 14-fold increase in the basal current (Figure 1F). Lower concentrations, however, also led to significant changes in the basal activity of hKCNK10, as this was increased by factors of $1.33 \pm 0.2$ and $2.25 \pm 0.4$ by the application of $100 \mu \mathrm{M}$ and $300 \mu \mathrm{M}$, respectively (Figure 1G). Because the highest concentration of 2-APB that was evaluated required a concentration of the vehicle compound DMSO higher than 1\% (3.3\% DMSO for $10 \mathrm{mM} 2$-APB), we evaluated the effect of this vehicle at its highest concentration used and compared this effect with both the basal current prior to application and basal current after the application of the same solution containing 2-APB (Figure 3B). We found no significant difference in the basal current prior and after the application of 3.3\% DMSO for $10 \mathrm{~s}$; this was true for both injected and uninjected oocytes (Figure 3A).

To determine whether this effect depended on the presence of any cytosolic factor, we proceeded to transfect HEK293 cells with hKCNK10 and measured cell-excised patches in the insideout configuration, exposing them to $3 \mathrm{mM} 2$-APB (1\% DMSO). Untransfected HEK293 showed no significant response to $3 \mathrm{mM}$ 2-APB (Figure 1H, left). Transfected HEK293 cells showed open channel noise, as previously described (Bang et al., 2000), which was markedly increased after the application of $3 \mathrm{mM} 2-$ $\mathrm{APB}$ (Figure 1H, right), demonstrating the direct nature of the interaction.

\section{2-APB ACTIVATES hKCNK2 AND hKCNK4 CHANNELS IN A DOSE-DEPENDENT MANNER}

We also investigated the effect of 2-APB on the other two members of the TREK subfamily. These are hKCNK2 (hTREK-1), which is the most thoroughly studied KCNK channel, and hKCNK4 (hTRAAK), which received its name because of its sensitivity to arachidonic acid, though it is also sensitive to other polyunsaturated fatty acids. Both channels are active at physiological body temperatures and have been shown to be necessary for appropriate thermoperception (Noël et al., 2009). Like KCNK10, they are expressed in both the central and peripheral human nervous system (Medhurst et al., 2001).

2-Aminoethoxydiphenyl borate induced dose-dependent and partially reversible activation of hKCNK2 and hKCNK4 in $X$. laevis oocytes expressing these channels (see Figure 2A). As for hKCNK10, 2-APB elicited more pronounced activation of these two channels at potentials above the $\mathrm{K}_{\mathrm{rev}}$ (Figure 2B). 2-APB had

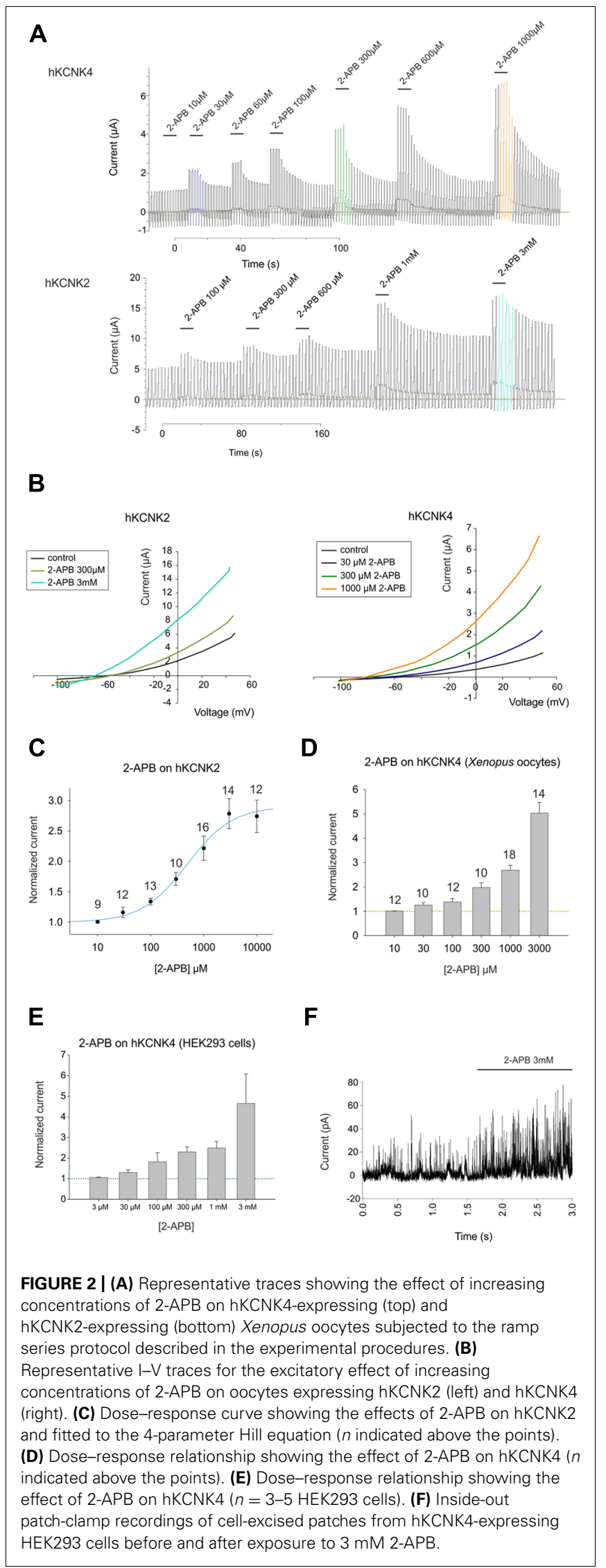

B
C

E
FIGURE 2 | (A) Representative traces showing the effect of increasing concentrations of 2-APB on hKCNK4-expressing (top) and series protocol described in the experimental procedures. (B) Representative I-V traces for the excitatory effect of increasing (C) Dose-response curve showing the effects of 2-APB on hi (D) Ditted to the 4-parameter Hill equation (n indicated above the points). indicated above the points). (E) Dose-response relationship showing the HEK293 cells before and after exposure to $3 \mathrm{mM}$ 2-APB. 
A

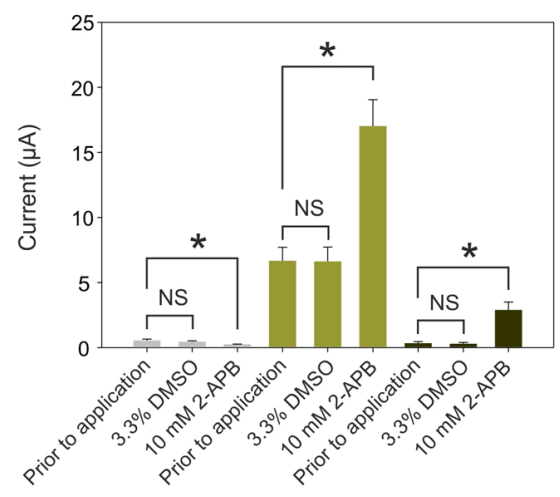

C

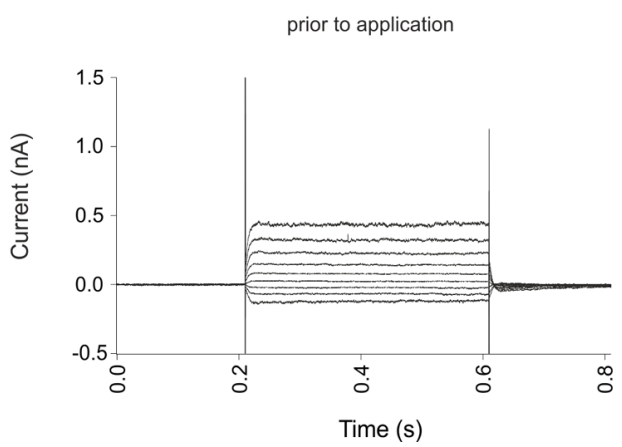

FIGURE 3 | (A) Comparison of the effects of applying $100 \mu \mathrm{L}$ frog Ringer's solution containing 3. 3\% DMSO vs. applying the same solution plus $10 \mathrm{mM}$ 2-APB to uninjected oocytes (gray bars) or oocytes injected with cRNA coding for hKCNK2 (light green bars) and hKCNK10 (dark green bars) $(n=3-5$ oocytes/group). The time of exposure was approximately $10 \mathrm{~s}$. (B) Top: Representative voltage clamp recording from an oocyte expressing hKCNK2 exposed to vehicle solution (green rectangles) or the same solution plus
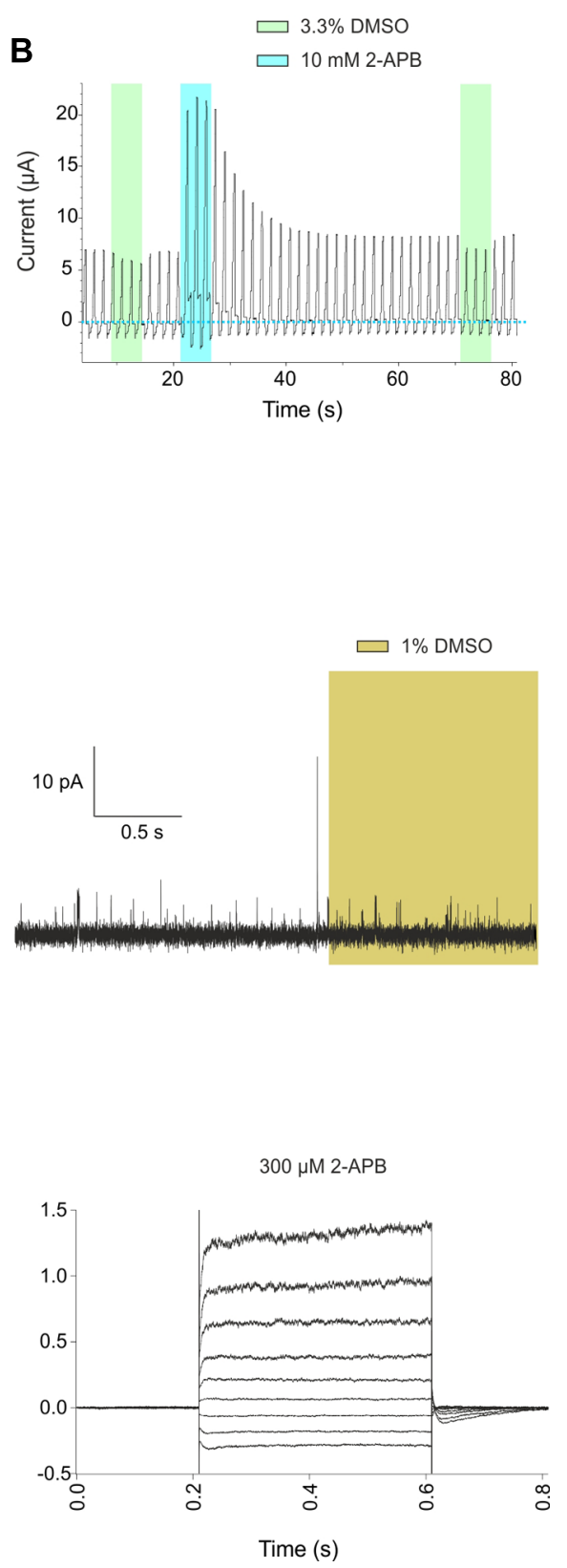

10 mM DMSO (blue rectangles). Bottom: Representative voltage clamp recording from an inside-out patch-clamp of a HEK293 cell expressing hKCNK10, before and after exposure to 1\% DMSO (light green rectangle). (C) hKCNK4 currents recorded from a transfected HEK293 cell before (right) and after (left) the application of $300 \mu \mathrm{M}$ 2-APB. Currents were elicited by voltage pulses from -110 to $+50 \mathrm{mV}$ in $20 \mathrm{mV}$ steps, $400 \mathrm{~ms}$ in duration, from a holding potential of $-60 \mathrm{mV}$. an $\mathrm{EC}_{50}$ of $486 \pm 135 \mu \mathrm{M}$ for hKCNK2 (Figure 2C); however, an $\mathrm{EC}_{50}$ value for hKCNK4 could not be determined, although this channel was clearly activated in a dose-dependent manner by 2APB in both X. laevis oocytes (Figure 2D) and transfected HEK293 cells (Figures 2E and 3C for original trace). Like hKCNK10, hKCNK4 also showed a marked increase in basal activity when treated with 2-APB in inside-out excised patches from transfected
HEK293 cells, suggesting that this effect is independent of cytosolic factors (Figure 2F).

\section{DISCUSSION}

Members of the TREK subfamily of KCNK channels are widely expressed in the CNS (Medhurst et al., 2001), where they have been shown to play a key role in several processes ranging from 
long-term depression to neuroprotection (Heurteaux et al., 2006; Kim etal., 2011). This makes them valuable targets for pharmacological research; however, little is known regarding their pharmacology, and common and stable activators are lacking. Here we have showed that the membrane-permeable substance 2-APB behaves as a highly effective activator for all the members of the mechano-gated TREK subfamily of human KCNK channels. 2-APB must now be regarded as a pharmacological tool for the study of members of the TREK subfamily. Also, its pharmacological characteristics might be useful for drug design. Interestingly, we also found that 2-APB can block certain unidentified endogenous channel(s) in X. laevis oocytes. This effect should be taken into consideration when using this system to evaluate 2 -APB at high concentrations, especially when characterizing the effects of 2-APB on heterologously expressed channels (e.g., TRPC and TRPM channels).

The basal activity of hKCNK10 was increased up to 2.3- and 14fold when exposed to $300 \mu \mathrm{M}$ and $3 \mathrm{mM} 2-\mathrm{APB}$, respectively. This is expected to significantly inhibit cell excitability, as observed in the Xenopus heterologous system, in which $1 \mathrm{mM}$ 2-APB induced a hyperpolarizing change in the membrane potential of around $-50 \mathrm{mV}$. It would be interesting to study the effect of this substance on native systems, e.g., trigeminal neurons. 2-APB also had considerable effects on the other members of the TREK subfamily, leading to a fourfold increase in their basal activities. However, as

\section{REFERENCES}

Alloui, A., Zimmermann, K., Mamet, J., Duprat, F., Noël, J., Chemin, J., et al. (2006). TREK-1, a K+ channel involved in polymodal pain perception. EMBO J. 25, 23682376.

Bang, H., Kim, Y., and Kim, D. (2000). TREK-2, a new member of the mechanosensitive tandem-pore K+ channel family. J. Biol. Chem. 275, 17412-17419.

Dobrydneva, Y., and Blackmore, P. (2001). 2-Aminoethoxydiphenyl borate directly inhibits storeoperated calcium entry channels in human platelets. Mol. Pharmacol. 3, 541-552.

Dörner, J. F., Gisselmann, G., Hatt, H., and Wetzel, C. H. (2007). Transient receptor potential channel A1 is directly gated by calcium ions. J. Biol. Chem. 282, 13180-13189.

Enyedi, P., and Czirják, G. (2010). Molecular background of leak $\mathrm{K}+$ currents: two-pore domain potassium channels. Physiol. Rev. 90, 559-605.

Goldstein, S., Bockenhauer, D., O’Kelly, I., and Zilberberg, N. (2001). Potassium leak channels and the KCNK family of two-P-domain subunits. Nat. Rev. Neurosci. 2, 175-184.

Gu, W., Schlichthörl, G., Hirsch, J. R., Engels, H., Karschin, C., Karschin, A., etal. (2002). Expression pattern and functional characteristics of two novel splice variants of the two-pore-domain potassium channel TREK-2. J. Physiol. 539, 657-668.

Heurteaux, C., Lucas, G., Guy, N., El Yacoubi, M., Thümmler, S., Peng, X. D., et al. (2006). Deletion of the background potassium channel TREK-1 results in a depression-resistant phenotype. Nat. Neurosci. 9, 1134 1141.

$\mathrm{Hu}$, H. Z., Gu, Q., Wang, C. Colton, C. K., Tang, J., KinoshitaKawada, M., et al. (2004). 2-Aminoethoxydiphenyl borate is a common activator of TRPV1, TRPV2, and TRPV3. J. Biol. Chem. 279, 35741-35748.

Kang, D., and Kim, D. (2006). TREK2 (K2P10.1) and TRESK (K2P18.1) are major background $\mathrm{K}+$ channels in dorsal root ganglion neurons. Am. J. Physiol. Cell Physiol. 291, C138C146.

Kim, E. J., Kang, D., and Han, J. (2011). Baicalein and wogonin are activators of rat TREK-2 two-pore domain $\mathrm{K}+$ channel. Acta Physiol. (Oxf.) 202, 185-192.

Lotshaw, D. P. (2007). Biophysical, pharmacological, and functional characteristics of cloned and native mammalian two-pore domain $\mathrm{K}+$ channels. Cell Biochem. Biophys. 47, 209-256.

Maruyama, T., Kanaji, T. Nakade, S., Kanno, T., and

stated, 2-APB acts upon a plethora of other proteins. For example, it activates members of the TRPV subfamily, inhibits members of the TRPC subfamily, and blocks sarco/endoplasmic reticulum $\mathrm{Ca}^{2+}$-ATPase pumps. Also complicating the interpretation is the fact that KCNK channels are known to be targets of several second messengers. Nevertheless, our work with excised patches clearly shows that the effect of 2-APB on the members of the TREK subfamily is independent of any cytosolic factor.

It is worth noticing that 2-APB has also been used in several systems (e.g., rat dorsal root ganglia) that endogenously express KCNK10 and KCNK2 as two of their major potassium background channels (Kang and Kim, 2006). Our results should be taken into consideration when using these systems to evaluate the effect of 2-APB at concentrations higher than $300 \mu \mathrm{M}$. At these concentrations, 2-APB increases by nearly a factor of 2 the basal activity of hKCNK4 and hKCNK10, while also significantly enhancing the basal activity of hKCNK2 (Figures 1G and 3C). Finally, elucidation of a possible common activating mechanism for members of this subfamily is deserving of further study.

\section{ACKNOWLEDGMENT}

This work was funded by a grant (SFB 874) from the German Research Foundation (Deutsche Forschungsgemeinschaft) to Hanns Hatt (TP A3).

Mikoshiba, K. (1997). 2APB, 2aminoethoxydiphenyl borate, a membrane-penetrable modulator of Ins $(1,4,5) \mathrm{P} 3$-induced $\mathrm{Ca} 2+$ release. J. Biochem. 122, 498-505.

Medhurst, A. D., Rennie, G., Chapman, C. G., Meadows, H., Duckworth, M. D., Kelsell, R. E., et al. (2001). Distribution analysis of human two pore domain potassium channels in tissues of the central nervous system and periphery. Brain Res. Mol. Brain Res. 86, 101-114.

Noël, J., Zimmermann, K., Busserolles, J., Deval, E., Alloui, A., Diochot, S. et al. (2009). The mechano-activated $\mathrm{K}+$ channels TRAAK and TREK-1 control both warm and cold perception. $E M B O$ J. 28, 1308-1318.

Vogt-Eisele, A. K., Weber, K., Sherkheli, M. A., Vielhaber, G., Panten, J., Gisselmann, G., et al. (2007). Monoterpenoid agonists of TRPV3. Br. J. Pharmacol. 151, 530-540.

Wang, Y., Deshpande, M., and Payne, R. (2002). 2-Aminoethoxydiphenyl borate inhibits phototransduction and blocks voltage-gated potassium channels in Limulus ven tral photoreceptors. Cell Calcium 4, 209-216.

Xian Tao, Li, Dyachenko, V., Zuzarte, M., Putzke, C., Preisig-Müller, R. Isenberg, G., etal. (2006). The stretch-activated potassium channel TREK-1 in rat cardiac ventricular muscle. Cardiovasc. Res. 69, 86-97.
Yamamoto, Y., Hatakeyama, T., and Taniguchi, K. (2009). Immunohistochemical colocalization of TREK-1, TREK-2 and TRAAK with TRP channels in the trigeminal ganglion cells. Neurosci. Lett. 454, 129-133.

Conflict of Interest Statement: The authors declare that the research was conducted in the absence of any commercial or financial relationships that could be construed as a potential conflict of interest.

Received: 03 July 2012; accepted: 24 April 2013; published online: 15 May 2013.

Citation: Beltrán L, Beltrán M, Aguado A, Gisselmann $G$ and Hatt $H$ (2013) 2-Aminoethoxydiphenyl borate activates the mechanically gated human KCNK channels KCNK 2 (TREK-1), KCNK 4 (TRAAK) and KCNK 10 (TREK-2). Front. Pharmacol. 4:63. doi: 10.3389/ fphar.2013.00063

This article was submitted to Frontiers in Neuropharmacology, a specialty of Frontiers in Pharmacology.

Copyright (c) 2013 Beltrán, Beltrán, Aguado, Gisselmann and Hatt. This is an open-access article distributed under the terms of the Creative Commons Attribution License, which permits use, distribution and reproduction in other forums, provided the original authors and source are credited and subject to any copyright notices concerning any thirdparty graphics etc. 